\title{
On eigenvalue inequalities of a matrix whose graph is bipartite
}

\author{
Abdullah Alazemi ${ }^{1 *}$, Milica Anđelić ${ }^{2}$ and Slobodan K. Simićc,3
}

"Correspondence: alazmi95@gmail.com

'Department of Mathematics, Kuwait University, Kuwait city, Kuwait

Full list of author information is available at the end of the article

\begin{abstract}
We consider the set of real zero diagonal symmetric matrices whose underlying graph, if not told otherwise, is bipartite. Then we establish relations between the eigenvalues of such matrices and those arising from their bipartite complement. Some accounts on interval matrices are provided. We also provide a partial answer to the still open problem posed in (Zhan in SIAM J. Matrix Anal. Appl. 27:851-860, 2006).

MSC: Primary 15A18; secondary 15A42; 05C50

Keywords: Bipartite complement of matrix; Eigenvalue bounds; Interlacing property; Interval matrices
\end{abstract}

\section{Introduction}

In this paper, we consider the eigenvalues of a real symmetric matrix of order $n \geq 2$ with zero diagonal. The set of such matrices is denoted by $\mathcal{M}_{n}$, that is,

$$
\mathcal{M}_{n}=\left\{A=\left(a_{i j}\right) \in \mathbb{R}^{n \times n}: a_{i j}=a_{j i}, a_{i i}=0\right\} .
$$

By $G(A)$ we denote the underlying graph of $A \in \mathcal{M}_{n}$, that is, it is a simple graph (without loops or multiple edges) whose vertices correspond to rows (or columns) of $A$ with two vertices $v_{i}$ and $v_{j}$ adjacent whenever the $(i, j)$ th entry of $A$ is nonzero. Let

$$
\mathcal{B}_{n}=\left\{A \in \mathcal{M}_{n}: G(A) \text { is bipartite }\right\} .
$$

We denote by $K_{n}$ the complete graph on $n$ vertices and by $K_{m, n}$ the complete bipartite graph with corresponding color classes of sizes $m, n$. We also use the following notation: $J_{n}$ denotes the all-ones matrix of order $n$, whereas $J_{m, n}$ is the all-ones matrix of order $m \times n$. Note that $A\left(K_{n}\right)=J_{n}-I_{n}$, where $I_{n}$ is the unit matrix of order $n$, whereas

$$
A\left(K_{m, n}\right)=\left[\begin{array}{cc}
O_{m} & J_{m, n} \\
J_{n, m} & O_{n}
\end{array}\right] .
$$

Throughout the paper, we assume that the eigenvalues of a matrix $A$, that is, the roots of its characteristic polynomial $\varphi_{A}(\lambda)=\operatorname{det}(\lambda I-A)$ (or, occasionally, its underlying graph $G=G(A))$ are ordered in nonincreasing way $\left(\lambda_{i}(A) \geq \lambda_{j}(A)\right.$ for $\left.i<j\right)$.

(c) The Author(s) 2019. This article is distributed under the terms of the Creative Commons Attribution 4.0 International License (http://creativecommons.org/licenses/by/4.0/), which permits unrestricted use, distribution, and reproduction in any medium, provided you give appropriate credit to the original author(s) and the source, provide a link to the Creative Commons license, and indicate if changes were made. 
We will frequently exploit the well-known Courant-Weyl inequalities (see, e.g., [2, p. 239]).

Theorem 1.1 Let $A$ and $B$ be $n \times n$ Hermitian matrices. Then

$$
\begin{array}{ll}
\lambda_{i}(A+B) \leq \lambda_{j}(A)+\lambda_{i-j+1}(B) & (n \geq i \geq j \geq 1), \\
\lambda_{i}(A+B) \geq \lambda_{j}(A)+\lambda_{i-j+n}(B) & (1 \leq i \leq j \leq n),
\end{array}
$$

with equality in (1.1) for a pair $i, j$ if and only if there is a nonzero vector $\mathbf{x}$ such that $A \mathbf{x}=$ $\lambda_{j}(A) \mathbf{x}, B \mathbf{x}=\lambda_{i-j+1}(B) \mathbf{x}$, and $(A+B) \mathbf{x}=\lambda_{i}(A+B) \mathbf{x}$ and in (1.2) if and only if there is a nonzero vector $\mathbf{x}$ such that $A \mathbf{x}=\lambda_{j}(A) \mathbf{x}, B \mathbf{x}=\lambda_{i-j+n}(B) \mathbf{x}$, and $(A+B) \mathbf{x}=\lambda_{i}(A+B) \mathbf{x}$. If $A$ and $B$ have no common eigenvector, then every inequality in (1.1) and (1.2) is strict.

The rest of the paper is organized as follows. In Sect. 2, we introduce the concept of a bipartite complement of a real symmetric matrix with zero diagonal whose underlying graph is bipartite, and then we show that the odd-indexed eigenvalues of the initial matrix interlace the even-indexed eigenvalues of its bipartite complement (and vice versa). In this section, we also show that the bounds on the second largest eigenvalue of matrices in $\mathcal{M}_{n}$ whose off-diagonal entries are in the (closed) interval [0,1], as given in [3], can be significantly improved in the case where the underlying graph of a matrix in question is bipartite. Also, we extend these results (i.e., bounds) in several directions. First, we prove that these bounds hold for all matrices in $\mathcal{M}_{n}$, not only for those with $a_{i j} \in[0,1]$; second, we prove that similar bounds hold for the other eigenvalues (not only for the second largest one). We also deduce, in the nonbipartite case, similar properties for a real symmetric matrix with zero diagonal with respect to its complement. In Sect. 3, we focus on matrices whose underlying graphs are bipartite and all nonzero entries are in a given interval. We provide both upper and lower bounds on the largest eigenvalue of these matrices. If the corresponding matrices are nonnegative, then we also provide upper and lower bounds on the second largest eigenvalue. Here we give a partial answer to the problem posed in [4], that is, we determine extremal values for the second largest eigenvalue of a real symmetric matrix whose entries lie in a nonnegative interval.

\section{Matrices with bipartite underlying graph}

Let $A \in \mathcal{B}_{n}$ and assume that $G(A)$ has two color classes of sizes $p$ and $q$. We write $\mathcal{B}_{p, q}$, $p+q=n, p, q>0$, if $p$ and $q$ are relevant further on. Then $A \in \mathcal{B}_{p, q}$ with suitable (and simultaneous) permutation of rows and columns has the following form:

$$
A=\left[\begin{array}{cc}
O_{p} & B \\
B^{T} & O_{q}
\end{array}\right]
$$

with $B \in \mathbb{R}^{p \times q}$. The bipartite complement of $A$ (with respect to the above form) is the matrix

$$
A^{b}=A\left(K_{p, q}\right)-A \text {, }
$$


where

$$
A\left(K_{p, q}\right)=\left[\begin{array}{cc}
O_{p} & J_{p, q} \\
J_{q, p} & O_{q}
\end{array}\right]
$$

that is, $A+A^{b}$ is the adjacency matrix of the complete bipartite graph $K_{p, q}$. Note that, for reducible symmetric matrices or, equivalently, for those with disconnected underlying graphs, such a representation (i.e., form) need not be unique. This stems from the fact that color classes only in disconnected bipartite graphs need not be uniquely determined. Note also that if we restrict ourselves to $\{0,1\}$-matrices or just simple graphs, then the bipartite complement of a disconnected bipartite graph need be neither unique nor connected (observe, for example, two copies of $\left.K_{1,2}\right)$. In the graph theory the bipartite complement may be also considered as a Seidel switching regarding the color classes (cf. [1, p. 5]). Finally, note that $\left(A^{b}\right)^{b}=A$, provided that color classes of $G(A)$ and $G\left(A^{b}\right)$ are preserved in both graphs.

We first establish relations between the eigenvalues of $A$ and $A^{b}$. Recall that the spectrum of a symmetric matrix with bipartite underlying graph is symmetric with respect to the origin (see, e.g., [1, p. 56]).

Theorem 2.1 Let $A \in \mathcal{B}_{p, q}(p+q \geq 3, p, q \geq 1)$, and let $A^{b}$ be its bipartite complement. If $n \geq 3$, then

$$
\lambda_{1}(A) \geq \lambda_{2}\left(A^{b}\right), \quad \lambda_{n}(A) \leq \lambda_{n-1}\left(A^{b}\right),
$$

and

$$
\lambda_{j+1}\left(A^{b}\right) \leq \lambda_{j}(A) \leq \lambda_{j-1}\left(A^{b}\right) \quad(2 \leq j \leq n-1) .
$$

Proof Since the eigenvalues of $A\left(K_{p, q}\right)$ are $\sqrt{p q}, \underbrace{0, \ldots, 0}_{n-2},-\sqrt{p q}$, by Theorem 1.1 (case (1.2)) we obtain

$$
0=\lambda_{2}\left(G\left(K_{p, q}\right)\right)=\lambda_{2}\left(A+A^{b}\right) \geq \lambda_{j}(A)+\lambda_{2-j+n}\left(A^{b}\right) \quad(2 \leq j \leq n) .
$$

Hence

$$
\lambda_{j}(A)+\lambda_{2-j+n}\left(A^{b}\right) \leq 0,
$$

that is, $\lambda_{j}(A) \leq-\lambda_{2-j+n}\left(A^{b}\right)$. Since the underlying graph of $A^{b}$ is bipartite, its spectrum is symmetric with respect to the origin, which implies $-\lambda_{2-j+n}\left(A^{b}\right)=\lambda_{j-1}\left(A^{b}\right)$ for all $2 \leq j \leq n$. So, we obtain

$$
\lambda_{j}(A) \leq \lambda_{j-1}\left(A^{b}\right) \quad(2 \leq j \leq n) .
$$

For $j=n$, we obtain the second inequality in (2.3). From (2.5), for $j \geq 2$, we can also obtain $\lambda_{n+2-j}\left(A^{b}\right) \leq-\lambda_{j}(A)=\lambda_{n+1-j}(A)$, which in turn is equivalent to $\lambda_{j}\left(A^{b}\right) \leq \lambda_{j-1}(A)$ for $2 \leq j \leq$ $n$. In particular, for $j=2$, we obtain the first inequality in (2.3). 
This result tells that the even- and odd-indexed eigenvalues of $A$ interlace the odd-and even-indexed eigenvalues of $A^{b}$, respectively. We now deduce when the first inequality in (2.3) becomes equality under the constraint that all entries of $A$ belong to [0,1].

Theorem 2.2 If $A \in \mathcal{B}_{p, q}(p+q \geq 3, p, q \geq 1)$ is a matrix with all entries in [0,1], then

$$
\lambda_{2}(A)=\lambda_{1}\left(A^{b}\right)
$$

if and only if $G\left(A^{b}\right)$ is disconnected, and either

$$
A=A\left(K_{p, q}\right) \quad \text { or, equivalently, } \quad A^{b}=O
$$

or there exists a nonzero vector $\mathbf{x} \in \mathbb{R}^{n}$ such that $A^{b} \mathbf{x}=\lambda_{n}\left(A^{b}\right) \mathbf{x}$ and

$$
(\underbrace{0, \ldots, 0}_{p}, \underbrace{1, \ldots, 1}_{q}) \mathbf{x}=0, \quad(\underbrace{1, \ldots, 1}_{p}, \underbrace{0, \ldots, 0}_{q}) \mathbf{x}=0 .
$$

Proof If $A=A\left(K_{p, q}\right)$, then we are immediately done. So let $A \neq A\left(K_{p, q}\right)$. Then

$$
\lambda_{2}(A)+\lambda_{n}\left(A^{b}\right)=\lambda_{2}\left(A\left(K_{p, q}\right)\right),
$$

since $\lambda_{n}\left(A^{b}\right)=-\lambda_{1}\left(A^{b}\right)$ and $\lambda_{2}\left(A\left(K_{p, q}\right)\right)=0$. By Theorem 1.1, case (1.2), the previous equality holds if and only if there exists a nonzero vector $\mathbf{x}$ such that

$$
A \mathbf{x}=\lambda_{2}(A) \mathbf{x}, \quad A^{b} \mathbf{x}=\lambda_{n}\left(A^{b}\right) \mathbf{x}, \quad A\left(K_{p, q}\right) \mathbf{x}=\lambda_{2}\left(A\left(K_{p, q}\right)\right) \mathbf{x}=\mathbf{0} .
$$

The last condition is equivalent to (2.7).

We now prove that $G\left(A^{b}\right)$ is disconnected. Suppose on the contrary that $G\left(A^{b}\right)$ is connected. Then $A^{b}$ is irreducible. If $\mathbf{x}=\left(\mathbf{y}^{t}, \mathbf{z}^{t}\right)^{t}$ is the eigenvector corresponding to $\lambda_{n}\left(A^{b}\right)$, then $\left(\mathbf{y}^{t},-\mathbf{z}^{t}\right)^{t}$ is the eigenvector corresponding to $\lambda_{1}\left(A^{b}\right)$. Therefore $\left(\mathbf{y}^{t},-\mathbf{z}^{t}\right)^{t}$ is a scalar multiple of the Perron eigenvector of $A^{b}$. (Note that both $A$ and $A^{b}$ are nonnegative.) This implies that all entries of $\mathbf{y}$ must be either strictly positive or strictly negative. Hence their sum cannot be equal to 0 as expected (see (2.7)). A similar argument holds for $\mathbf{z}$, and we arrive at a contradiction.

Suppose now that there exists a nonzero vector $\mathbf{x}$ such that $A^{b} \mathbf{x}=\lambda_{n}\left(A^{b}\right) \mathbf{x}$ and (2.7) holds. Since $A+A^{b}=A\left(K_{p, q}\right)$, we have $\left(A+A^{b}\right) \mathbf{x}=A\left(K_{p, q}\right) \mathbf{x}$ and $A \mathbf{x}+A^{b} \mathbf{x}=\mathbf{0}$, since $A\left(K_{p, q}\right) \mathbf{x}=\mathbf{0}$ due to (2.7). Therefore $A \mathbf{x}=-\lambda_{n}\left(A^{b}\right) \mathbf{x}=\lambda_{1}\left(A^{b}\right) \mathbf{x}$. This means that $\lambda_{1}\left(A^{b}\right)$ is the eigenvalue of $A$, and we only need to prove that it is not equal to $\lambda_{1}(A)$. Since (2.7) holds, $\mathbf{x}$ cannot be a Perron vector, because all entries of a Perron vector can be taken to be nonnegative.

This completes the proof.

Remark 2.1 By applying Courant-Weyl inequalities to the largest eigenvalue of $A+A^{b}$, that is, to $\lambda_{1}\left(A\left(K_{p, q}\right)\right)$, we obtain $\lambda_{1}(A)+\lambda_{1}\left(A^{b}\right) \geq \sqrt{p q}$.

Example 2.1 If $G$ is a nearly complete bipartite graph (with only a few edges missing) and $A_{G}$ is its adjacency matrix, then the bound (2.3) can be very good. For example, for 
$G=K_{5,6}-e, \lambda_{2}\left(A_{G}\right)=0.84076$, and $\lambda_{1}\left(A_{G}^{b}\right)=1$. For $G=K_{5,6}-e_{1}-e_{2}$, where $e_{1}$ and $e_{2}$ are two nonadjacent edges in $K_{5,6}, \lambda_{2}\left(A_{G}\right)=\lambda_{1}\left(A_{G}^{b}\right)=1$.

Next, we consider the Kronecker product $A\left(K_{2}\right) \otimes A$ for any matrix $A \in \mathcal{M}_{n}$. Then

$$
\left[\begin{array}{ll}
0 & 1 \\
1 & 0
\end{array}\right] \otimes A=\left[\begin{array}{ll}
O & A \\
A & O
\end{array}\right],
$$

which means that the underlying graph of $A\left(K_{2}\right) \otimes A$ is bipartite. The eigenvalues of $A\left(K_{2}\right) \otimes A$ are $\pm \lambda_{i}(A)$ for $1 \leq i \leq n$ (see [1, p. 44]). The bipartite complement of $A\left(K_{2}\right) \otimes A$ has the following form:

$$
\left(A\left(K_{2}\right) \otimes A\right)^{b}=\left[\begin{array}{cc}
O & A^{c}+I_{n} \\
A^{c}+I_{n} & O
\end{array}\right],
$$

where $A^{c}=J_{n}-I_{n}-A$ (the underlying graph of $A+A^{c}$ is $K_{n}$ ). In view of Theorems 2.1 and 2.2 and previous observations, together with he corresponding inequalities (2.3) and (2.4) applied to both $A$ and $A^{c}$, we have the following:

Theorem 2.3 Let $A$ be a real symmetric matrix with zero diagonal, and let $A^{c}=J_{n}-I_{n}-A$ be its complement. If $n \geq 2$, then

$$
\lambda_{1}(A) \geq \lambda_{2}\left(A^{c}\right)+1, \quad \lambda_{n}(A) \leq \lambda_{n-1}\left(A^{c}\right)-1,
$$

and

$$
\lambda_{j+1}\left(A^{c}\right)+1 \leq \lambda_{j}(A) \leq \lambda_{j-1}\left(A^{c}\right)-1 \quad(2 \leq j \leq n-1) .
$$

If $A$ is a matrix with all entries in $[0,1]$ and if $G\left(\left(A\left(K_{2}\right) \otimes A\right)^{b}\right)$ is disconnected, then

$$
\lambda_{2}(A)=\lambda_{1}\left(A^{c}\right)-1
$$

if and only if either $A=J_{n}-I_{n}$ or, equivalently, $A^{c}=O$, or there exists a nonzero vector $\mathbf{x} \in \mathbb{R}^{n}$ such that $A^{c} \mathbf{x}=\lambda_{n}\left(A^{c}\right) \mathbf{x}$ and $\mathbf{j}^{t} \mathbf{x}=0$.

Remark 2.2 Theorem 2.3 generalizes Proposition 2.1 from [3] since it provides interlacing between all the eigenvalues of $A$ (i.e., $A^{c}$ ), not only between the largest and second largest ones.

We conclude this section with the following result.

Proposition 2.4 Let $A=\left[\begin{array}{cc}O & B \\ B^{t} & O\end{array}\right] \in \mathcal{B}_{p, q}$ be an irreducible nonnegative matrix such that the matrix $B$ has the fixed row (column) sum equal to $r$ (resp., $s$ ). Then $\sigma(A)$, the spectrum of $A$, is

$$
\pm \sqrt{r s}, \quad \pm \mu_{2}{ }^{m_{2}}, \quad \ldots, \quad \pm \mu_{k}{ }^{m_{k}}
$$




$$
\text { - } \begin{aligned}
& \sigma\left(A^{b}\right)= \pm(\sqrt{p q}-\sqrt{r s}), \pm \mu_{2}^{m_{2}}, \ldots, \pm \mu_{k}^{m_{k}} \text { if } \sqrt{p q}-\sqrt{r s} \neq \lambda_{2}(A), \\
& \text { - } \sigma\left(A^{b}\right)= \pm(\sqrt{p q}-\sqrt{r s})^{m_{2}+1}, \pm \mu_{3}^{m_{3}}, \ldots, \pm \mu_{k}^{m_{k}} \text { if } \sqrt{p q}-\sqrt{r s}=\lambda_{2}(A) .
\end{aligned}
$$

Proof Since the matrices $A$ and $A\left(K_{p, q}\right)$ are symmetric and commute, they can be simultaneously diagonalized by, for example, matrix $P$, that is, $P^{-1} A P=D_{1}$ and $P^{-1} A\left(K_{p, q}\right) P=D$. Consequently, $P^{-1} A^{b} P=D-D_{1}$. The matrix $A\left(K_{p, q}\right)$ has three distinct eigenvalues, namely $\sqrt{p q}, 0,-\sqrt{p q}$ with multiplicities $1, p+q-2,1$, respectively. Also, $\lambda_{1}(A)=\sqrt{\lambda_{1}\left(B B^{t}\right)}=\sqrt{r s}$, since $B B^{t}$ has the constant row sum equal to $r s$. Now the result directly follows.

\section{Interval matrices}

By an interval matrix we assume a matrix whose all entries lie in some interval, say (closed) interval $[a, b](-\infty<a<b<+\infty)$. Its diagonal entries in general need not be equal to 0 . Let $\mathcal{S}_{n}[a, b]$ denote the set of all symmetric interval matrices over the interval $[a, b]$. In [4] the range of extremal eigenvalues of a real symmetric interval matrix was considered.

Here we consider the set of symmetric matrices whose all nonzero entries lie in the interval $[a, b]$ and whose underlying graphs are bipartite with bipartition $U \cup V$, where $|U|=p,|V|=q, p+q=n, p, q>0$. Without loss of generality we may assume that this set, say $\mathcal{B}_{p, q}^{\prime}[a, b]$, consists of all matrices of the form $\left[\begin{array}{cc}O & B \\ B^{t} & O\end{array}\right]$, where $B \in[a, b]^{p \times q}$ for some $p+q=n, p, q>0$.

We first determine $\max \left\{\lambda_{1}(A): A \in \mathcal{B}_{p, q}^{\prime}[a, b]\right\}$ and $\min \left\{\lambda_{1}(A): A \in \mathcal{B}_{p, q}^{\prime}[a, b]\right\}$, and we also identify matrices that attain these extremal values.

Let $A=\left[\begin{array}{cc}O & B \\ B^{t} & O\end{array}\right] \in \mathcal{B}_{p, q}^{\prime}[a, b]$. Without loss of generality, we assume that $p \geq q$. Then $A^{2}=$ $\left[\begin{array}{cc}B B^{t} & O \\ O & B^{t} B\end{array}\right]$ and $\varphi_{A^{2}}(\lambda)=\lambda^{p-q} \varphi_{B^{t} B}^{2}(\lambda)$. Hence $\lambda_{1}(A)=\sqrt{\lambda_{1}\left(B^{t} B\right)}$, where

$$
B^{t} B \in \begin{cases}S_{q}\left[p a^{2}, p b^{2}\right], & 0 \leq a<b ; \\ S_{q}\left[p b^{2}, p a^{2}\right], & a<b \leq 0 ; \\ S_{q}\left[p a b, p b^{2}\right], & a \leq 0<b,|a| \leq b ; \\ S_{q}\left[p a b, p a^{2}\right], & a<0 \leq b,|a|>b .\end{cases}
$$

Next, we apply the following results to $B^{t} B$.

Theorem 3.1 ([4]) Let $A \in \mathcal{S}_{n}[a, b]$ with $n>2$ and $a<b$.

(i) If $|a|>b$, then

$$
\lambda_{1}(A) \leq \begin{cases}n(b-a) / 2 & \text { if } n \text { is even } \\ \left(n b-\sqrt{b^{2}+\left(n^{2}-1\right) a^{2}}\right) / 2 & \text { if } n \text { is odd }\end{cases}
$$

If $n$ is even, then the equality holds if and only if $A$ is permutation similar to

$$
\left[\begin{array}{ll}
b J_{\frac{n}{2}} & a J_{\frac{n}{2}} \\
a J_{\frac{n}{2}} & b J_{\frac{n}{2}}
\end{array}\right]
$$

If $n$ is odd, then the equality holds if and only if $A$ is permutation similar to

$$
\left[\begin{array}{cc}
b J_{\frac{n-1}{2}} & a J_{\frac{n-1}{2}, \frac{n+1}{2}} \\
a J_{\frac{n+1}{2}, \frac{n-1}{2}} & b J_{\frac{n+1}{2}}
\end{array}\right] .
$$


(ii) If $|a| \leq b$, then $\lambda_{1}(A) \leq n b$. If $|a|<b$, then the equality holds if and only if $A=b J_{n}$.

If $|a|=b$, then the equality holds if and only if $A$ is permutation similar to

$$
\left[\begin{array}{cc}
b J_{k} & a J_{k, n-k} \\
a J_{n-k, k} & b J_{n-k}
\end{array}\right]
$$

for some $k$ with $1 \leq k \leq n$.

Theorem 3.2 ([4]) Let $A \in \mathcal{S}_{n}[a, b]$ with $n>2$.

- If $0<a<b$, then $\lambda_{1}(A) \geq n a$ with equality if and only if $A=a J_{n}$.

- If $a \leq 0<b$, then $\lambda_{1}(A) \geq a$ with equality if and only if $A=a I_{n}$.

According to the intervals where entries of $B^{t} B$ belong to, we distinguish the following cases:

(1) If $0<a<b$, then $\lambda_{1}(A) \leq \sqrt{p q} b$. The equality holds if and only if $B=b J_{p q}$. Also, $\lambda_{1}(A) \geq \sqrt{p q} a$. The equality holds if and only if $B=a J_{p q}$.

(2) If $a<b<0$, then $\lambda_{1}(A) \leq \sqrt{p q}|a|$. The equality holds if and only if $B=a J_{p q}$. Here $\lambda_{1}(A) \geq \sqrt{p q}|b|$. The equality holds if and only if $B=b J_{p q}$.

(3) If $a \leq 0<b,|a| \leq b$, then $\lambda_{1}(A) \leq \sqrt{p q} b$. If $|a|=b$, then the equality holds if and only if $B$ is permutation similar to

$$
\left[\begin{array}{cc}
b J_{k} & a J_{k, p-k} \\
a J_{p-k, k} & b J_{p-k}
\end{array}\right]
$$

for some $k$ with $1 \leq k \leq n$. If $|a|<b$, then the holds if and only if $B=b J_{p q}$. In this case, $\lambda_{1}(A) \geq 0$. The equality holds if and only if $B=O_{p q}$, since $B^{t} B$ is positive semidefinite.

(4) If $a<0 \leq b,|a|>b$, then $\lambda_{1}(A) \leq \sqrt{p q}|a|$. The equality holds if and only if $B=a J_{p q}$. Also, $\lambda_{1}(A) \geq 0$. The equality holds if and only if $B=O_{p q}$.

Next, we determine upper and lower bounds on the second largest eigenvalue of bipartite interval matrices with given cardinality of color classes in the case where $0 \leq a<b$ or $a<b \leq 0$.

Recall first the following:

Theorem 3.3 ([2, p. 238]) Let $A \in \mathbb{R}^{n \times n}$ be a symmetric matrix, and let $\lambda_{1}(A) \geq \cdots \geq \lambda_{n}(A)$ be its eigenvalues given in nonincreasing fashion. Let $S$ be a subspace of $\mathbb{R}^{n}$. Then

$$
\lambda_{2}(A)=\min _{\left\{S \leq \mathbb{R}^{n}: \operatorname{dim} S=n-1\right\}} \max _{\{\mathbf{x}: \mathbf{x} \in S,\|\mathbf{x}\|=1\}} \mathbf{x}^{t} A \mathbf{x} .
$$

Next, we provide an upper bound for the second largest eigenvalue of a nonnegative matrix with entries in a given interval.

Theorem 3.4 Let $A \in \mathcal{S}_{n}[a, b], n \geq 2,0 \leq a<b$, be an irreducible matrix. Then

$$
\lambda_{2}(A) \leq \begin{cases}n(b-a) / 2 & \text { if } n \text { is even }, \\ \left(n b-\sqrt{b^{2}+\left(n^{2}-1\right) a^{2}}\right) / 2 & \text { if } n \text { is odd }\end{cases}
$$


Proof Let $\mathbf{v}_{1}, \mathbf{v}_{2}$ be unit eigenvectors corresponding to $\lambda_{1}(A)$ and $\lambda_{2}(A)$, respectively. Then $\mathbf{v}_{2}$ is orthogonal to $\mathbf{v}_{1}$. Since $A$ is a nonnegative matrix, all entries of $\mathbf{v}_{1}$ can be taken to be nonnegative (see [2, p. 529]). Due to orthogonality, $\mathbf{v}_{2}$ has both nonnegative and negative entries. By simultaneous row and column permutations of $A$, if necessary, we may assume that the first $k$ entries of $\mathbf{v}_{2}$ are nonnegative, whereas the remaining are negative for some $k<n$. Without loss of generality, we may also assume that $k \geq n-k$. (In the opposite case, instead of $\mathbf{v}_{2}$, we would consider $-\mathbf{v}_{2}$.) Also, $\lambda_{2}(A)$ can be written in the form

$$
\lambda_{2}(A)=\mathbf{v}_{2}^{t} A \mathbf{v}_{2}=\mathbf{j}^{t}\left[A \circ \mathbf{v}_{2} \mathbf{v}_{2}^{t}\right] \mathbf{j}
$$

where $\circ$ denotes the Hadamard entrywise product of matrices, and $\mathbf{j}$ is the all-ones vector. Note that $A \circ \mathbf{v}_{2} \mathbf{v}_{2}^{t}$ has the sign pattern $\left[\begin{array}{cc}{ }^{+} k & -k, n-k \\ -n-k, k & { }_{n-k}\end{array}\right]$. Let

$$
\hat{A}=J(k, a, b)=\left[\begin{array}{cc}
b J_{k} & a J_{k, n-k} \\
a J_{n-k, k} & b J_{n-k}
\end{array}\right]
$$

and

$$
\mathbb{R}_{k, n-k}^{n}=\left\{\left(x_{1}, \ldots, x_{n}\right)^{t} \in \mathbb{R}^{n}: x_{1}, \ldots, x_{k} \geq 0, x_{k+1}, \ldots, x_{n}<0\right\} .
$$

For any $\mathbf{x} \in \mathbb{R}_{k, n-k}^{n}, \mathbf{x}^{t} A \mathbf{x} \leq \mathbf{x}^{t} \hat{A} \mathbf{x}$, and thus

$$
\begin{aligned}
& \max _{\left\{\mathbf{x}: \mathbf{x} \in S \cap \mathbb{R}_{k, n-k}^{n},\|\mathbf{x}\|=1\right\}, \operatorname{dim} S=n-1} \mathbf{x}^{t} A \mathbf{x} \leq \max _{\left\{\mathbf{x}: \mathbf{x} \in S \cap \mathbb{R}_{k, n-k}^{n},\|\mathbf{x}\|=1\right\}, \operatorname{dim} S=n-1} \mathbf{x}^{t} \hat{A} \mathbf{x}, \\
& \min _{\left\{S \leq \mathbb{R}^{n}: \operatorname{dim} S=n-1\right\}\left\{\mathbf{x}: \mathbf{x} \in S \cap \mathbb{R}_{k, n-k^{n}}^{n},\|\mathbf{x}\|=1\right\}} \mathbf{x}^{t} A \mathbf{x} \leq \min _{\left\{S \leq \mathbb{R}^{n}: \operatorname{dim} S=n-1\right\}\left\{\mathbf{x}: \mathbf{x} \in S \cap \mathbb{R}_{k, n-k^{n}}^{n},\|\mathbf{x}\|=1\right\}} \mathbf{x}^{t} \hat{A} \mathbf{x} .
\end{aligned}
$$

Since both $A$ and $\hat{A}$ have at least one eigenvector corresponding to $\lambda_{2}(A)$, that is, $\lambda_{2}(\hat{A})$ belonging to $\mathbb{R}_{k, n-k}^{n}$, by (3.2) it follows

$$
\begin{aligned}
\lambda_{2}(A) & =\min _{\left\{S \leq \mathbb{R}^{n}: \operatorname{dim} S=n-1\right\}} \max _{\mathbf{x} \in S \cap \mathbb{R}_{k, n-k}^{n},\|\mathbf{x}\|=1} \mathbf{x}^{t} A \mathbf{x} \\
& \leq \min _{\left\{S \leq \mathbb{R}^{n}: \operatorname{dim} S=n-1\right\}} \max _{\left\{\mathbf{x}: \mathbf{x} \in S \cap \mathbb{R}_{k, n-k}^{n},\|\mathbf{x}\|=1\right\}} \mathbf{x}^{t} \hat{A} \mathbf{x}=\lambda_{2}(\hat{A}),
\end{aligned}
$$

that is,

$$
\lambda_{2}(A) \leq \lambda_{2}(J(k, a, b))
$$

Therefore the maximal value for $\lambda_{2}(A)$ is attained at some matrix of the form (3.4). The eigenvalues of $\hat{A}$ are 0 with multiplicity $n-2$, and $\lambda_{1,2}(\hat{A})=\frac{n b \pm \sqrt{(n-2 k) b^{2}+4 k(n-k) a^{2}}}{2}$. Since $0 \leq a<b$,

$$
\lambda_{2}(\hat{A})=\frac{n b-\sqrt{(n-2 k) b^{2}+4 k(n-k) a^{2}}}{2}>0 .
$$

If $n$ is even, then the right-hand side of (3.6) attains its maximum at $k=\frac{n}{2}$, and then $\lambda_{2}(A) \leq$ $\lambda_{2}\left(J\left(\frac{n}{2}, a, b\right)\right)=\frac{n}{2}(b-a)$. 
If $n$ is odd, then the maximal value in (3.6) is attained at $k=\frac{n-1}{2}$ or $k=\frac{n+1}{2}$. Hence

$$
\lambda_{2}(A) \leq \lambda_{2}\left(J\left(\frac{n-1}{2}, a, b\right)\right)=\frac{n b-\sqrt{b^{2}+\left(n^{2}-1\right) a^{2}}}{2}
$$

This completes the proof.

Theorem 3.5 Let $A \in \mathcal{S}_{n}[a, b], n \geq 2,0 \leq a<b$, be an irreducible matrix. If $n$ is even, then $\lambda_{2}(A)=\frac{n}{2}(b-a)$ if and only if $A$ is permutation similar to

$$
\left[\begin{array}{ll}
b J_{\frac{n}{2}} & a J_{\frac{n}{2}} \\
a J_{\frac{n}{2}} & b J_{\frac{n}{2}}
\end{array}\right]
$$

If $n$ is odd, then $\lambda_{2}(A)=\frac{1}{2}\left(n b-\sqrt{b^{2}+\left(n^{2}-1\right) a^{2}}\right)$ if and only if $A$ is permutation similar to

$$
\left[\begin{array}{cc}
b J_{\frac{n-1}{2}} & a J_{\frac{n-1}{2}, \frac{n+1}{2}} \\
a J_{\frac{n+1}{2}, \frac{n-1}{2}} & b J_{\frac{n+1}{2}}
\end{array}\right] .
$$

Proof For even $n$, let $A \in \mathcal{S}_{n}[a, b]$ be a matrix satisfying $\lambda_{2}(A)=\frac{n}{2}(b-a)$, and let $\mathbf{x}=$ $\left(x_{1}, \ldots, x_{n}\right)^{t}$ be a corresponding unit eigenvector. First, suppose that $\mathbf{x}$ has no zero entries and that $k$ of them are positive and $n-k$ negative. If $k \neq \frac{n}{2}$, then by (3.5) we know that $\lambda_{2}(A) \leq \lambda_{2}(J(k, a, b))<\lambda_{2}\left(J\left(\frac{n}{2}, a, b\right)\right)=\frac{n(b-a)}{2}$, a contradiction. Therefore $k=\frac{n}{2}$. By simultaneous row and column permutations of $A$, if necessary, we may assume that $x_{i}>0$ for $i \leq \frac{n}{2}$ and $x_{j}<0$ for $j>\frac{n}{2}$. Then

$$
\lambda_{2}(A)=\mathbf{x}^{t} A \mathbf{x} \leq \mathbf{x}^{t} J\left(\frac{n}{2}, a, b\right) \mathbf{x} \leq \lambda_{2}(A)
$$

implies that $A=J\left(\frac{n}{2}, a, b\right)$. Otherwise, the first inequality would be strict, a contradiction. Therefore, the initial matrix $A$ is permutation similar to $J\left(\frac{n}{2}, a, b\right)$.

The equality condition for odd $n$ when $\mathbf{x}$ has no zero components can be proved similarly, and therefore we omit it. Note that

$$
\left[\begin{array}{cc}
b J_{\frac{n+1}{2}} & a J_{\frac{n+1}{2}, \frac{n-1}{2}} \\
a J_{\frac{n-1}{2}, \frac{n+1}{2}} & b J_{\frac{n-1}{2}}
\end{array}\right] \text { and }\left[\begin{array}{cc}
b J_{\frac{n-1}{2}} & a J_{\frac{n-1}{2}, \frac{n+1}{2}} \\
a J_{\frac{n+1}{2}, \frac{n-1}{2}} & b J_{\frac{n+1}{2}}
\end{array}\right]
$$

are permutation similar.

Otherwise, if $n$ is even and $\mathbf{x}$ has zero entries, then without loss of generality it can be represented in the form $\mathbf{x}=(\underbrace{0, \ldots, 0}_{n-k^{\prime}})$, where $\mathbf{u} \in \mathbb{R}^{k^{\prime}}, k^{\prime}<n$, and $\mathbf{u}$ has no zero entries. Consequently, $A$ can be partitioned in the form $\left[\begin{array}{cc}\tilde{A} & B \\ B^{t} & C\end{array}\right]$, where $\tilde{A} \in \mathbb{R}^{k^{\prime} \times k^{\prime}}, B \in \mathbb{R}^{k^{\prime} \times\left(n-k^{\prime}\right)}$, and $C \in \mathbb{R}^{\left(n-k^{\prime}\right) \times\left(n-k^{\prime}\right)}$, provided that

$$
\begin{aligned}
& \tilde{A} \mathbf{u}=\lambda_{2}(A) \mathbf{u}, \\
& B^{t} \mathbf{u}=0
\end{aligned}
$$


From (3.7) it follows that $\lambda_{2}(A) \in \operatorname{Spec}(\tilde{A})$, and therefore $\lambda_{2}(A)=\lambda_{1}(\tilde{A})$ or $\lambda_{2}(A)=\lambda_{2}(\tilde{A})$. If the second equality holds, then $\mathbf{u}$ is the corresponding eigenvector for $\lambda_{2}(\tilde{A})$ with no zero entries, and hence from the first case it follows that $\lambda_{2}(A)=\lambda_{2}(\tilde{A}) \leq \frac{k^{\prime}}{2}(b-a)<\frac{n}{2}(b-a)$ if $k^{\prime}$ is even and similarly if $k^{\prime}$ is odd, a contradiction.

Therefore $\lambda_{2}(A)=\lambda_{1}(\tilde{A})$, and $\mathbf{u}$ is the corresponding positive eigenvector (since we assumed that $\mathbf{u}$ has no zero entries). From (3.8) it follows that any row of $B^{t}$ is orthogonal to $\mathbf{u}$, and consequently $B^{t}=O$ since $B^{t}$ is a nonnegative matrix (note that $a=0$ in this case). Assume that $\mathbf{v}_{1}=\left[\mathbf{v}^{t}, \mathbf{w}^{t}\right], \mathbf{v} \in \mathbb{R}^{k^{\prime}}, \mathbf{w} \in \mathbb{R}^{n-k^{\prime}}$. Having in mind that $B=O$, it easily follows that $C \mathbf{w}=\lambda_{1}(A) \mathbf{w}$, that is, $\lambda_{1}(A) \in \operatorname{Spec}(C)$, which implies $\lambda_{1}(A)=\lambda_{1}(C)$.

According to Theorem 3.1, if $n$ is even and $k^{\prime}<\frac{n}{2}$, then $\lambda_{2}(A)=\lambda_{1}(\tilde{A}) \leq k^{\prime} b<\frac{n}{2} b$, a contradiction. Hence $k^{\prime}=\frac{n}{2}$. Moreover, again by Theorem 3.1, $\lambda_{1}(\tilde{A})=\frac{n}{2} b$ if and only if $\tilde{A}=b J_{\frac{n}{2}}$. Consequently, $\frac{n}{2} b=\lambda_{2}(A) \leq \lambda_{1}(A) \leq\left(n-k^{\prime}\right) b=\frac{n}{2} b$, that is, $\lambda_{1}(C)=\frac{n}{2} b$, which implies $C=b J_{\frac{n}{2}}$. Hence $A=J\left(\frac{n}{2}, 0, b\right)$.

If $n$ is odd and $\mathbf{x}$ contains zeros, in a similar way, we can prove that the equality holds if and only if $A=J\left(\frac{n-1}{2}, 0, b\right)$.

This completes the proof.

For the remaining cases, where not both $a$ and $b$ are nonnegative, we conjecture the following:

\section{Conjecture 1 Let}

$$
\hat{A}(r, s, t)=\left[\begin{array}{ccc}
b J_{r} & a J_{r s} & a J_{r t} \\
a J_{s r} & b J_{s} & a J_{s t} \\
a J_{t r} & a J_{t s} & b J_{t}
\end{array}\right], \quad \tilde{B}(k, l)=\left[\begin{array}{cc}
b J_{k} & O_{k l} \\
O_{l k} & b J_{l}
\end{array}\right] \text {, }
$$

and let $A \in \mathcal{S}_{n}[a, b], n \geq 2$, be an irreducible matrix.

(i) If $a<0 \leq b$, then

$$
\lambda_{2}(A) \leq \max _{\substack{r+s+t=n, k+l=n, r, s, t, k, l \geq 1}}\left\{\lambda_{2}(\hat{A}(r, s, t)), \lambda_{2}(\tilde{B}(k, l))\right\}
$$

(ii) If $a<b \leq 0$, then

$$
\lambda_{2}(A) \leq \max _{r+s+t=n, r, s, t \geq 1} \lambda_{2}(\hat{A}(r, s, t)) .
$$

For $A=\left[\begin{array}{cc}O & B \\ B^{t} & O\end{array}\right]$, where $B \in \mathbb{R}^{p \times q}[a, b], B^{t} B$ is nonnegative if $0 \leq a<b$ or $a<b \leq 0$. Since $\lambda_{2}(A)=\sqrt{\lambda_{2}\left(B^{t} B\right)}$, it follows that

$$
\lambda_{2}(A) \leq \begin{cases}\sqrt{\frac{p q\left|b^{2}-a^{2}\right|}{2}} & \text { if } q \text { is even and } 0 \leq a<b \text { or } a<b \leq 0, \\ \sqrt{\frac{p q b^{2}-\sqrt{p^{2} b^{4}+\left(q^{2}-1\right) p^{2} a^{4}}}{2}} & \text { if } q \text { is odd and } 0 \leq a<b, \\ \sqrt{\frac{p q a^{2}-\sqrt{p^{2} a^{4}+\left(q^{2}-1\right) p^{2} b^{4}}}{2}} & \text { if } q \text { is odd and } a<b \leq 0 .\end{cases}
$$

We next consider the lower bounds for second largest eigenvalue of the matrix in $\mathcal{S}_{n}[a, b]$ for $|a|<b$. 
Recall that $\lambda_{2}(A) \geq \lambda_{2}(C)$, where $C$ is any principal submatrix of $A$ of order $k$. In particular,

$$
\lambda_{2}(A) \geq \lambda_{2}\left(\left[\begin{array}{ll}
a_{i i} & a_{i j} \\
a_{j i} & a_{j j}
\end{array}\right]\right)=\frac{a_{i i}+a_{j j}-\sqrt{\left(a_{i i}-a_{j j}\right)^{2}+4 a_{i j}^{2}}}{2} \geq a-b .
$$

Thus $\lambda_{2}(A) \geq a-b$. If $\lambda_{2}(A)=a-b$, then all the inequalities in (3.9) must be equalities. This forces $a_{i i}=a_{j j}=a$ and $a_{i j}=a_{j i}=b$. As this should be true for all $i<j, A=b J+(a-b) I$.

Therefore we proved the following:

Theorem 3.6 Let $A \in \mathcal{S}_{n}[a, b], n \geq 2,|a|<b$, then

$$
\lambda_{2}(A) \geq a-b .
$$

The equality holds if and only if $A=b J+(a-b) I$.

Next, we focus only on bipartite interval matrices belonging to the set $\mathcal{B}_{p, q}^{\prime}[0,1]$.

Proposition 3.7 Let $A \in \mathcal{B}_{p, q}^{\prime}[0,1], n \geq 3$. Then $\lambda_{2}(A) \leq q / 2$.

Proof Let $d_{i}(A)$ be the row sum of $i$ th row of $A, D=\operatorname{diag}\left(d_{1}(A), \ldots, d_{n}(A)\right)$, and $Q=D+A=$ $(D-A)+2 A$. The matrix $D-A$ is positive semidefinite, and therefore $\lambda_{2}(A) \leq \lambda_{2}(Q) / 2$ (see [2, p. 495]). On the other hand, $\left[\begin{array}{cc}q I_{p} & J_{p q} \\ J_{q p} & p I_{q}\end{array}\right]=Q+\left(\left[\begin{array}{cc}q I_{p} & I_{p q} \\ J_{q p} & p I_{q}\end{array}\right]-Q\right)$, and $\left[\begin{array}{cc}q I_{p} & I_{p q} \\ J_{q p} & p I_{q}\end{array}\right]-Q$ is pos-

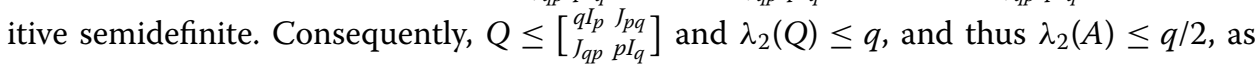
required.

Proposition 3.8 Let $A \in \mathcal{B}_{p, q}^{\prime}[0,1]$. Then

$$
\lambda_{2}(A) \leq \max \{q-r(B), p-s(B)\},
$$

where $r(B)$ and $s(B)$ are the minimal row and column sums in $B$, respectively.

Proof The bound is a direct consequence of Theorem 2.1 and the Frobenius upper bound:

$$
\lambda_{2}(A) \leq \lambda_{1}\left(A^{b}\right) \leq \max _{1 \leq i \leq n} r_{i}\left(A^{b}\right)=\max \{q-r(B), p-s(B)\} .
$$

\section{Acknowledgements}

The authors acknowledge the support of the Research Sector, Kuwait University. The authors would like to thank the anonymous referees for careful reading and helpful comments and suggestions that lead to the improvement of the original manuscript.

Funding

Not applicable.

Competing interests

The authors declare that they have no competing interests.

Authors' contributions

All three authors contributed equally in writing this manuscript. They all read and approved the final version.

\section{Author details}

${ }^{1}$ Department of Mathematics, Kuwait University, Kuwait city, Kuwait. ${ }^{2}$ ENEL, D.O.O., Belgrade, Serbia. ${ }^{3}$ Mathematical Institute SANU, Belgrade, Serbia. 


\section{Publisher's Note}

Springer Nature remains neutral with regard to jurisdictional claims in published maps and institutional affiliations.

Received: 30 October 2018 Accepted: 18 February 2019 Published online: 27 February 2019

\section{References}

1. Cvetković, D., Rowlinson, S., Simić, S.: An Introduction to the Theory of Graph Spectra. Cambridge University Press, New York (2010)

2. Horn, R.A., Johnson, C.R.: Matrix Analysis. Cambridge University Press, Cambridge (2013)

3. Kolotilina, L.Y.: Upper bounds for the second largest eigenvalue of symmetric nonnegative matrices. J. Math. Sci. 191, 75-89 (2013)

4. Zhan, X.: Extremal eigenvalues of real symmetric matrices with entries in an interval. SIAM J. Matrix Anal. Appl. 27, 851-860 (2006)

Submit your manuscript to a SpringerOpen ${ }^{\circ}$ journal and benefit from:

- Convenient online submission

- Rigorous peer review

- Open access: articles freely available online

- High visibility within the field

- Retaining the copyright to your article

Submit your next manuscript at $\gg$ springeropen.com 